\title{
A MzScheme Implementation of Transition P Systems
}

\author{
Delia Balbontín Noval, Mario J. PÉrez Jiménez and \\ Fernando SANCHO CAPARRINI \\ Dpto. Ciencias de la Computación e Inteligencia Artificial \\ Universidad de Sevilla, España \\ \{Delia.Balbontin, Mario.Perez, Fernando. Sancho\}@cs.us.es
}

\begin{abstract}
The main goal of this paper is to present the design of an MzScheme program that allows us to simulate the behavior of transition $\mathrm{P}$ systems. For that, a library of procedures have been developed that work in two stages. In the first one, the parsing/compiling stage, the input $\mathrm{P}$ system is checked, and if it is well defined, then it is represented by means of an internal grammar. In a second stage, the simulation, the computation tree associated to the $\mathrm{P}$ system is generated until a prefixed level.
\end{abstract}

\section{Introduction}

In October 1998, Gheorghe Păun [?] introduced a new computability model of a non-deterministic and highly parallel type, the membrane systems. They are based on the synchronized work of several units, called membranes, structured in a dynamic hierarchy (understood as vesicles in a space) embedded in a skin membrane that separates the system from the environment. When a membrane has no membrane inside, it is called elementary. Each membrane encloses a space between it and the membranes directly included in it (if any). This space (the region of the membrane) can contain a multiset (a set where the elements can be repeated) of objects (represented by symbols of a given alphabet) and a set of (evolution) rules for them. Each membrane defines an unique region.

This model, called transition P systems, is inspired from the observation that the processes which take place in the complex structure of a living cell can be viewed as computation-like processes.

We present here a library of MzScheme procedures [?], that allows us both to input easily a transition $\mathrm{P}$ system and to simulate its non-deterministic and highly parallel behavior. It reads, analyzes and compiles the input data defining a $\mathrm{P}$ system; then, it generates the subsequent computations.

Our implementation is based on the formalization given in [?].

The program runs in two independent stages: parsing/compiling and simulation/running.

$$
\Pi \text {-input } \stackrel{\text { parser/compiler }}{\longrightarrow} \Pi \stackrel{\text { simulator }}{\longrightarrow} \operatorname{Comp}(\Pi)
$$


At stage one, parsing/compiling, the input data are read and the respective $\mathrm{P}$ system is rewritten as an element of the language generated by a proposed internal grammar. To get it, the input data have to be syntactically correct according to the input grammar. Moreover, they have to define a well defined $\mathrm{P}$ system (according to the formalization above mentioned).

Stage two, simulation/running, starts when the parsing/compiling is finished. Starting from the $\mathrm{P}$ system initial configuration, the associated computation tree is generated. The expansion of that computation tree is made in a progressive way, level by level (breadth expansion), until to a given depth level. To get it we follow a breadth-expansion-tree scheme based on the definitions and steps proposed in [?]:

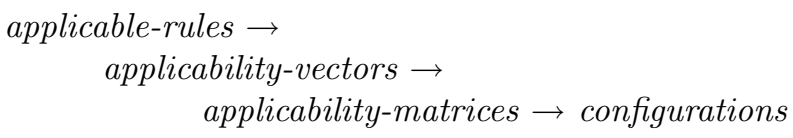

This paper is organized as follows: Section 2 briefly presents some basic concepts about a formalization of transition $\mathrm{P}$ systems, following [?]. Section 3 describes briefly the whole simulator scheme. Section 4 is about the way to input a $\mathrm{P}$ system, showing the proposed input grammar. Section 5 presents the internal grammar and describes the parser/compiler performance. Section 6 describes the simulator behavior properly. Finally, in Section 7 we present a complete example to illustrate the way of working of the program.

\section{Preliminaries about a formalization of transition $\mathbf{P}$ systems}

Following [?], we recall here the basic concepts and definitions about $\mathrm{P}$ systems.

\subsection{Membrane structure and cells}

A membrane structure is a rooted tree, where the nodes are called membranes, the root is called skin, and the leaves are called elementary membranes.

A cell over an alphabet, $A$, is a pair $(\mu, M)$, where $\mu=(V(\mu), E(\mu))$ is a membrane structure, and $M$ is an application, $M: V(\mu) \longrightarrow \mathbf{M}(A)$ (the set of multisets over $A$ ).

\subsection{Evolution rules}

Let $C=(\mu, M)$ be a cell over an alphabet $A$. Let $x \in V(\mu)$. An evolution rule associated to $x$ is a 3-tuple $r=\left(d_{r}, v_{r}, \delta_{r}\right)$ where $d_{r}$ is the left-side of the rule, $v_{r}$ is the right-side of the rule, and $\delta_{r} \in\{\neg \delta, \delta\}$ indicates if the application of the rule dissolves the membrane.

A collection $R$ of evolution rules associated to $C$ is a function with domain $V(\mu)$ such that for every membrane $x \in V(\mu), R_{x}=\left\{r_{x, 1}, \ldots, r_{x, s_{x}}\right\}$ is a finite 
set (possibly empty) of (evolution) rules associated to $x$. A priority relation over $R$ is a function, $\rho$, with domain $V(\mu)$ such that for every membrane $x \in V(\mu)$, $\rho_{x}$ is a strict partial order over $R_{x}$ (possibly empty).

\subsection{Transition $\mathbf{P}$ systems}

A transition $P$ system is a 4 -tuple $\Pi=\left(A, C_{0}, \mathcal{R}, i_{0}\right)$, where:

- $A$ is a non-empty finite set (usually called base alphabet).

- $C_{0}=\left(\mu_{0}, M_{0}\right)$ is a cell over $A$.

- $\mathcal{R}$ is an ordered pair $(R, \rho)$ where $R$ is a collection of (evolution) rules associated to $C_{0}$, and $\rho$ is a priority relation over $R$.

- $i_{0}$ is a node of $\mu_{0}$, which specifies the output membrane of $\Pi$.

The number $\left|V\left(\mu_{0}\right)\right|$ is called the degree of $\Pi$.

\subsection{Configurations}

A configuration, $C$, of a $\mathrm{P}$ system, $\Pi=\left(A, C_{0}, \mathcal{R}, i_{0}\right)$ with $C_{0}=\left(\mu_{0}, M_{0}\right)$, is a cell $C=(\mu, M)$ over $A$, where $V(\mu) \subseteq V\left(\mu_{0}\right)$, and $\mu$ has the same root as $\mu_{0}$. The configuration $C_{0}$ will be called the initial configuration of $\Pi$.

\subsection{Applicability}

Let $x \in V\left(\mu_{0}\right)$. We say that the (evolution) rule $r \in R_{x}$ is semi-applicable to $C$ if the membrane associated to node $x$ exists in $C$ (dissolution is not allowed in the root node), the membrane associated to $x$ has all the necessary objects to apply the rule, and nodes where the rule tries to send objects (by means of $i n_{y}$ ) are children of $x$.

We say that the rule $r \in R_{x}$ is applicable to $C$, if it is semi-applicable to $C$ and there is no semi-applicable rules in $R_{x}$ with higher priority.

We say that $\boldsymbol{p} \in \mathbf{N}^{\mathbf{N}}$ is an applicability vector over $x \in V(\mu)$ for $C$, and we will denote it as $\boldsymbol{p} \in \mathbf{A p}(x, C)$, if it has correct size (that is, for all $j$ greater the number of rules associated to $x$ we have $p(j)=\emptyset$ ), every rule can be applied as many times as the vector $\boldsymbol{p}$ indicates, all the rules can be applied simultaneously, and it is maximal.

We will say that $P: V\left(\mu_{0}\right) \longrightarrow \mathbf{N}^{\mathbf{N}}$ is an applicability matrix over $C$, denoted $P \in \mathbf{M}_{\mathbf{A} \mathbf{p}}(C)$, if for every $x \in V\left(\mu_{0}\right)$ we have that $P(x) \in \mathbf{A p}(x, C)$.

\subsection{Transitions}

The execution of $P$ over $C=(\mu, M)$, denoted $P(C)$, returns a new configuration $C^{\prime}=\left(\mu^{\prime}, M^{\prime}\right)$ of $\Pi$, that can be considered acting in two stages: $(\mu, M) \rightarrow$ $\left(\mu, M^{\prime \prime}\right) \rightarrow\left(\mu^{\prime}, M^{\prime}\right)$.

In the first stage we suppose that the rules are applied without attending dissolving actions, and in the second one dissolution and distribution of contents are carried out. 
We will say that a configuration $C_{1}$ of a $P$ system $\Pi$ yields a configuration $C_{2}$ by a transition in one step of $\Pi$, denoted $C_{1} \Rightarrow_{\Pi} C_{2}$, if there exists a non-zero applicability matrix over $C_{1}, P$, such that $P\left(C_{1}\right)=C_{2}$.

\subsection{Computation tree}

The computation tree of a $P$ system $\Pi$, denoted $\operatorname{Comp}(\Pi)$, is a rooted labeled maximal tree defined as follows: the root of the tree is the initial configuration, $C_{0}$, of $\Pi$; the children of a node are the configurations that follow in one step of transition; nodes and edges are labeled by configurations and applicability matrices, respectively, in such way that two labeled nodes $C, C^{\prime}$ are adjacent in $\operatorname{Comp}(\Pi)$, by means of an edge labeled with $P$, if and only if $P \in \mathbf{M}_{\mathbf{A p}}(C)-\{\mathbf{0}\}$ and $C^{\prime}=P(C)$. The maximal branches of $\operatorname{Comp}(\Pi)$ will be called computations of $\Pi$. We will say that a computation of $\Pi$ halts if it is a finite branch. The configurations verifying $\mathbf{M}_{\mathbf{A p}}(C)=\{\mathbf{0}\}$ will be called halting configurations.

\section{Preliminaries about the $\mathbf{P}$ systems simulator}

We consider that the basic features of a computing program able to simulate transition $\mathrm{P}$ systems should be the following:

1. To have a formal definition of transition $\mathrm{P}$ systems to be based on.

2. To choose a suitable programming language to implement the simulation.

3. To have an easy way to input the data describing the $\mathrm{P}$ system.

4. To choose an efficient internal representation of $\mathrm{P}$ systems.

5. To design a parser/compiler to analyze the input data and to obtain the $\mathrm{P}$ system internal representation.

6. To design a $\mathrm{P}$ system simulator of computations to generate the respective computation tree.

As we said previously, the implementation we present here has been developed on MzScheme (a functional language from Lisp family), and it is based on the formalization given in the above section, but slightly modified. This modification arises from the convenience to identify the applicable rules to a given configuration.

The rules of a $\mathrm{P}$ system are static elements. Nevertheless, to determine if a rule $r=\left(d_{r}, v_{r}, \delta_{r}\right)$ is applicable to an arbitrary configuration $C$, a new component $\alpha_{r} \in\{\# \mathrm{t}, \# \mathrm{f}\}$ has been added, getting $r^{*}=\left(d_{r}, v_{r}, \delta_{r}, \alpha_{r}\right)$. Initially, $\alpha_{r}$ will be set to \#f; it will be modified to \#t if (and only if) the rule $r$ is applicable to $C$. Consequently, if we denoted for every $x \in V\left(\mu_{0}\right), R_{x}=\left\{r_{x, 1}, \ldots, r_{x, s_{x}}\right\}$, then we have $R_{x}^{*}=\left\{r_{x, 1}^{*}, \ldots, r_{x, s_{x}}^{*}\right\}$, with $r_{x, j}^{*}=\left(d_{x, j}, v_{x, j}, \delta_{x, j}, \alpha_{x, j}\right)$ and $\alpha_{x, j}=\# \mathrm{f}$; then, $R^{*}=\bigcup_{x \in V\left(\mu_{0}\right)} R_{x}^{*}$, and $\mathcal{R}=\left(R^{*}, \rho\right)$.

Moreover, for every configuration, $C=(\mu, M)$ and every $x \in V\left(\mu_{0}\right)$, we will denote by $R_{x}^{C}=\left\{r_{x, 1}^{C}, \ldots, r_{x, s_{x}}^{C}\right\}$, with $r_{x, j}^{C}=\left(d_{x, j}, v_{x, j}, \delta_{x, j}, \alpha_{x, j}\right)$ and $\alpha_{x, j}=\# \mathrm{t}$ if and only if the rule $r_{x, j}$ is applicable to $C$, the tagged-rules of $x$ to $C$. Finally, we will note $R^{C}=\bigcup_{x \in V\left(\mu_{0}\right)} R_{x}^{C}$. 


\section{The Input of a Transition $P$ system}

To define a $\mathrm{P}$ system we need to input its membrane structure and describe the content of every membrane. Each membrane has symbols from a given alphabet, transition rules and priority relations over them. The membrane structure has to be a rooted tree and the priority between rules must be a strict partial order.

\subsection{Default Settings}

In order to introduce easily any $\mathrm{P}$ system we have considered, by default, that:

1. Only finite alphabets $A$ will be used, and the elements of $A$ are symbols.

2. A word $\in A^{*}$ is a string of symbols of $A$. We will represent the empty word by () .

3. The membranes will be labeled with the the first $N$ natural numbers, where $N$ is the degree of the $\mathrm{P}$ system.

4. The skin membrane is labeled with 1 .

5. A distinguished membrane is considered as the output membrane.

6. We will input the membrane structure of a $\mathrm{P}$ system as a list of contain-pairs $(i j)$, representing the relation "membrane $i$ contains membrane $j$ ".

7. Every rule has a word as its antecedent, and a set of actions as its consequent. Only the last action could be "delete". The other ones have the form (word target).

8. A target could be "here", "out" or a membrane label.

9. If a membrane has $k>0$ rules, then their labels go from 1 to $k$.

10. We represent the relation "rule $r$ runs before rule $s$ " by the preference-pair $(r s)$.

11. Every membrane contains a word, a list of rules, and a list of preference-pairs.

\subsection{The input grammar}

With the default settings provided above, any $\mathrm{P}$ system of degree $N$, over an alphabet $A$, is recognized by the input grammar defined as follows:

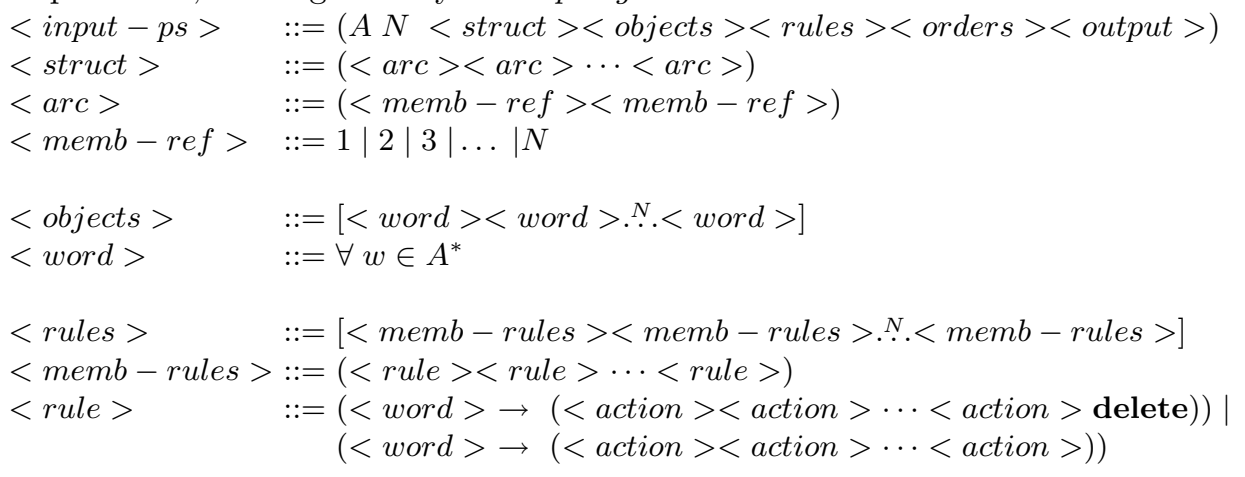




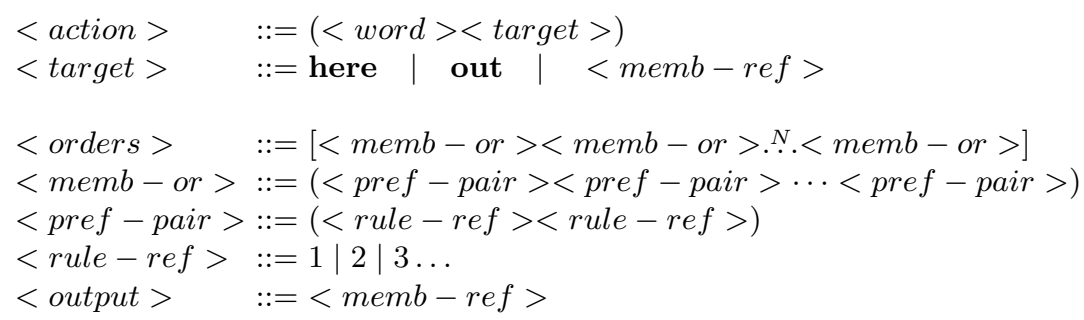

Here, $(a b \ldots z)$ stands for a list (standard MzScheme list), and $[a b \stackrel{N}{N}) z]$ stands for a vector of $N$ elements (standard MzScheme vector).

\section{The parser/compiler}

The parser/compiler reads the input data describing a $\mathrm{P}$ system and analyzes: if they are syntactically correct according to the input grammar, if they define a well defined $\mathrm{P}$ system according to the chosen formalization, and, if no error appears, it returns the $\mathrm{P}$ system according to the proposed internal grammar.

Even if the input system is syntactically correct, we cannot conclude that any input data recognized by the input grammar, define a well-defined $\mathrm{P}$ system. In fact, it could happen that the structure $<$ struct $>$ defined as a list of arcs $\left.(<\operatorname{arc}\rangle^{*}\right)$ were not a rooted tree with root at membrane label 1 ; or, that there exists a membrane, such that the order relation $(<$ mem - or $\rangle)$ defined as a list of preference pairs $\left(<\right.$ pref - pair $\left.>^{*}\right)$ were not a strict partial order.

The MzScheme sentence to execute the parser/compiler is:

(parser-ps $N A$ <struct> <objects> <rules> <orders> <output>)

This process of parsing/compiling works as follows:

- The alphabet $A$ is checked.

- The rooted tree $\mu$, associated to the membrane structure, is created.

- For every membrane $x$, its objects are encoded as a multiset $M_{x}$, getting $M: V(\mu) \longrightarrow \mathbf{M}(A)$.

- Then, the initial configuration, $C_{0}=(\mu, M)$, is built.

- Every rule, $r$, from the input data is encoded by $r^{*}=\left(d_{r}, v_{r}, \delta_{r}, \alpha_{r}\right)$, where $\alpha_{r}$ is set initially to $\# \mathrm{f}$. Then, one gets $R^{*}$.

- For every membrane $x$ a strict partial order $\rho_{x}: R_{x} \times R_{x} \longrightarrow\{\# \mathrm{t}, \# \mathrm{f}\}$ is returned, with: $\rho_{x}(r, t)=\# \mathrm{t} \Leftrightarrow$ " $r$ runs before $s$ " at $x$. So, we obtain $\rho$.

- From $R^{*}$ and $\rho$ we have $\mathcal{R}=\left(R^{*}, \rho\right)$.

- The output membrane is checked to be in $V(\mu)$, getting $i_{0}$.

If no error occurs, the parser-ps procedure returns a well-defined $\mathrm{P}$ system $\Pi=\left(A, C_{0}, \mathcal{R}, i_{0}\right)$ as an element recognized by the internal grammar below. 


\subsection{Internal Grammar}

The grammar to represent internally and to deal with $\mathrm{P}$ systems of degree $N$ is the following:

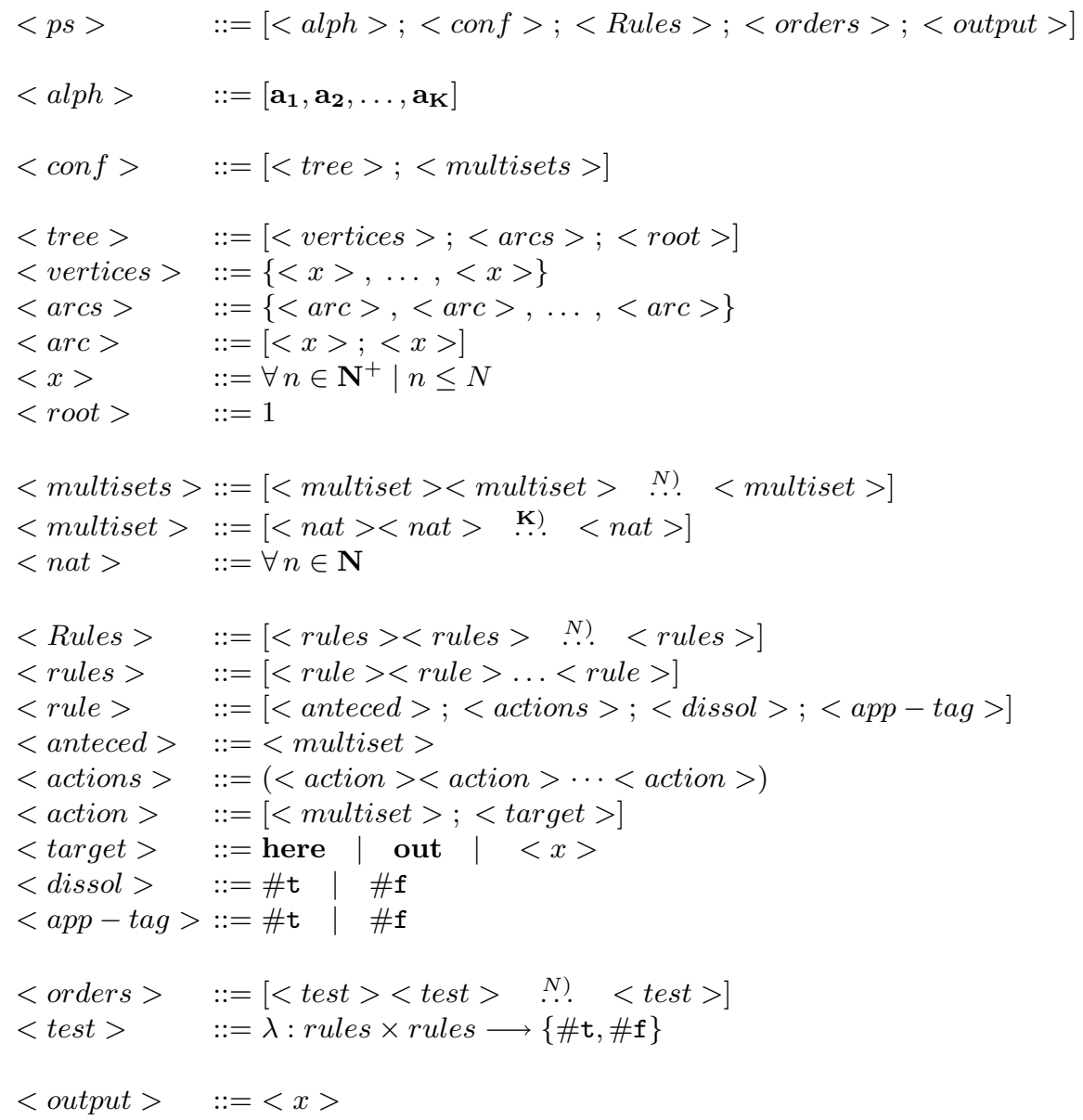

\section{The simulator}

Once the parsing/compiling task is finished, we have a well-defined $\mathrm{P}$ system, namely $\Pi=\left(A, C_{0}, \mathcal{R}, i_{0}\right)$, and we have to generate the computation tree $\operatorname{Comp}(\Pi)$. To do that we use the procedure configurations:

\section{$\Pi \stackrel{\text { configurations }}{\longrightarrow} \operatorname{Comp}(\Pi)$}

We get the computation tree $\operatorname{Comp}(\Pi)$ through the MzScheme sentence (configurations $\Pi$ level). 
The procedure configurations is based on the breadth-expansion-tree procedure that, starting from the initial configuration $C_{0}$, generates level by level the computation tree. It needs the auxiliary procedures applicability-vectors, tag-rules and apply-matrix. Here we present a brief outline. We will give in the next sections a detailed description of every one.

The operators to compute the successor configurations of a given configuration, $C$, are the applicability matrices. The process to generate the elements of $\mathbf{M}_{\mathbf{A}_{\mathbf{P}}}(C)$ works as follows:

- $R^{C}$ (that is, the tagged-rules of $x$ to $C$ ) is obtained by the tag-rules procedure. For every rule $r^{*}=\left(d_{r}, v_{r}, \delta_{r}, \alpha_{r}\right) \in R^{*}$, it sets $\alpha_{r}=\# \mathrm{t}$ iff $r$ is applicable to $C$.

- Every $R_{x}^{C}$, for every membrane $x$ in $C$, is easily obtained from $R^{C}$.

- Every $\operatorname{Ap}(x, C)$ (that is, the applicability vectors of membrane $x$ in $C$ ) is generated from $R_{x}^{C}$, by means of the applicability-vectors procedure.

- Finally, $\mathbf{M}_{\mathbf{A p}}(C)$ is constructed as a cartesian product from the set of applicability vectors $\mathbf{A p}(x, C)$, of every membrane $x$ in $C$.

$$
\begin{aligned}
& { }^{x_{1}} \quad R_{x_{1}}^{C} \stackrel{\text { applicability-vectors }}{\longrightarrow} \mathbf{A p}\left(x_{1}, C\right) \searrow \\
& C \stackrel{\text { tag-rules }}{\longrightarrow} R^{C \stackrel{\mathrm{x}_{2}}{\longrightarrow}} R_{x_{2}}^{C} \stackrel{\text { applicability-vectors }}{\longrightarrow} \mathbf{A p}\left(x_{2}, C\right) \rightarrow \mathbf{M}_{\mathbf{A}_{\mathbf{P}}}(C) \\
& R_{x_{3}}^{x_{3}} \stackrel{\text { applicability-vectors }}{\longrightarrow} \mathbf{A p}\left(x_{3}, C\right) \nearrow
\end{aligned}
$$

Then, every $P \in \mathbf{M}_{\mathbf{A p}}(C)$ is applied to $C$ to obtain the successor configuration $P(C)$. To do that the apply-matrix procedure is used.

$$
\begin{array}{r}
\nearrow P_{1} \in \mathbf{M}_{\mathbf{A}_{\mathbf{P}}}(C) \stackrel{\text { apply-matrix }}{\longrightarrow} C_{1}=P_{1}(C) \\
\mathbf{M}_{\mathbf{A}_{\mathbf{P}}}(C) \rightarrow P_{2} \in \mathbf{M}_{\mathbf{A}_{\mathbf{P}}}(C) \stackrel{\text { apply-matrix }}{\longrightarrow} C_{2}=P_{2}(C) \\
\searrow P_{3} \in \mathbf{M}_{\mathbf{A}_{\mathbf{P}}}(C) \stackrel{\text { apply-matrix }}{\longrightarrow} C_{3}=P_{3}(C)
\end{array}
$$

\subsection{The breadth-expansion-tree procedure}

This procedure is based on a dynamic breadth-search scheme; this means that for every node of the tree to be built, the applicable operators are generated dynamically.

To start, the breadth-expansion-tree procedure needs: (1) an initial node, $n_{0},(2)$ a test final-node? to check if a node $n$ is or not a final node, (3) a function generate-op, that, taking a node $n$, returns the set of operators $O p_{n}$ to be applied to $n$ and, finally, (4) another function apply-op that, taking a node $n$ and an operator $o p \in O p_{n}$, returns the successor node of $n$ by this operator op.

The breadth-expansion-tree procedure expands the tree and returns the set of final nodes. 


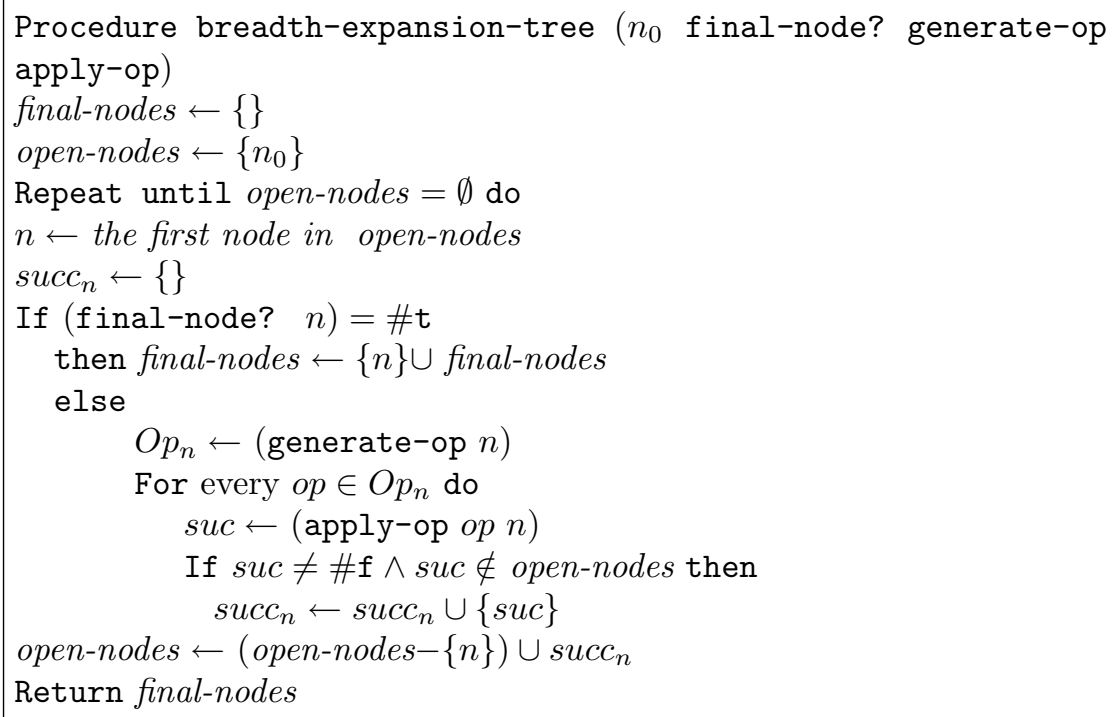

The procedures configurations and applicability-vectors, to generate configurations and applicability vectors, respectively, are based on this procedure.

\subsection{The configurations procedure}

For a given $\mathrm{P}$ system $\Pi=\left(A, C_{0}, \mathcal{R}, i_{0}\right)$, we generate $\operatorname{Comp}(\Pi)$ (until a level given by the user), through the MzScheme sentence (configurations $\Pi$ level). This procedure works as follows:

1. It starts defining locally:

- The node-structure as $<$ node $>::=\left[C ; R^{C} ;\right.$ path $\left._{C}\right]$, where $C$ ia a configuration; $R^{C}$, the tagged-rules for $C$; and path $C$, the list of operators applied to reach the actual node from the initial one.

- The final-node? test. A node $n=\left[C ; R^{C}\right.$,path $]$ is a final node if either it is a halting node, or the path length has reached the value of level.

- The generate-op function. It takes a node $n=\left[C ; \mathbf{R}^{C} ;\right.$ path $]$ and returns the applicability matrices $\mathbf{M}_{\mathbf{A p}}(C)$. It needs the procedure applicability-vectors.

- Finally, the procedure apply-op, which, taking a node $n=\left[C ; R^{C}\right.$,path $]$ and an applicability matrix $P \in \mathbf{M}_{\mathbf{A p}}(C)$, returns the successor node $n^{\prime}=\left[C^{\prime} ; R^{C^{\prime}} ; P \cup\right.$ path $]$. It needs the procedures apply-matrix and tag-rules.

2. Then, it builds the init-node: $n_{0}=\left[C_{0} ; R^{C_{0}} ;()\right]$, making use of the procedure tag-rules to get $R^{C_{0}}$. 
3. It expands the tree through the sentence:

(breadth-expansion-tree

init - node final-node? generate-op apply-op)

4. Finally, it returns the list of final-nodes $\left[C ; R^{C} ;\right.$ path $\left._{C}\right]$.

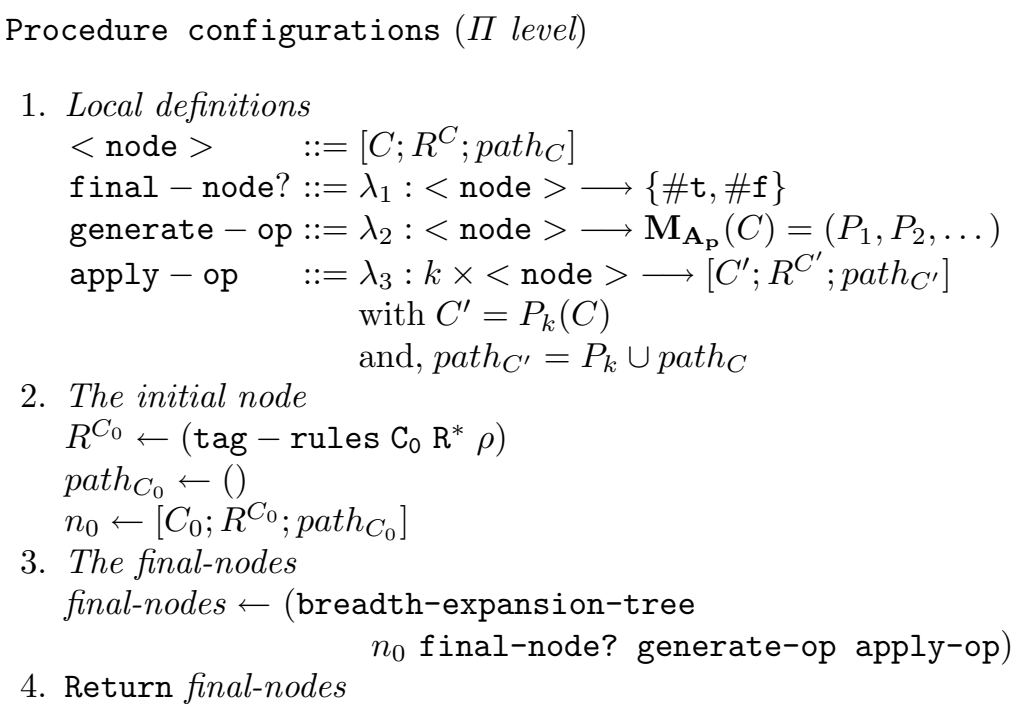

2. The initial node and, path $_{C^{\prime}}=P_{k} \cup$ path $_{C}$

3. The final-nodes final-nodes $\leftarrow$ (breadth-expansion-tree

4. Return final-nodes

Notes:

$-\lambda_{1}\left(\left[C ; R^{C} ;\right.\right.$ path $\left.\left.h_{C}\right]\right)=\# \mathrm{t} \leftrightarrow\left(\alpha_{r}=\# \mathrm{f} \forall r \in R^{C}\right) \vee \mid$ path $h_{C} \mid=$ level

- $\lambda_{2}$ uses applicability-vectors procedure to get $\mathbf{M}_{\mathbf{A}_{\mathbf{p}}}(C)$.

$-\lambda_{3}$ uses apply-matrix procedure to get $C^{\prime}=P(C)$ and then, tag-rules to get $R^{C^{\prime}}$.

- Every node $\left[C ; R^{C} ;\right.$ path $\left._{C}\right] \in$ final-nodes, contains all the information we need about the computation tree. Particularly,

- If for every $r \in R^{C}$ is $\alpha_{r}=\# \mathrm{f}$, then $C$ is a halting configuration, and path $_{C}$ is a halting computation of $\Pi$.

- Otherwise, $C$ is a non-halting configuration, and the branch path ${ }_{C}$ could be extended further than the prefixed level.

\subsection{The applicability-vectors procedure}

To generate the applicability vectors for a membrane $x$ in $C$, we only need $M_{x}$ and $D=\left[d_{1}, d_{2}, \ldots, d_{s_{x}}\right]$, where $M_{x}$ is the multiset of $x$, and $d_{r}\left(r=1,2, \ldots, s_{x}\right)$ is the antecedent of the tagged-rule $r$ in $R_{x}^{C}$, provided that $\alpha_{r}=\# \mathrm{t}$. (Note: if $\alpha_{r}=\# \mathbf{f}$, then we take $d_{r}=\# \mathbf{f}$.) We generate $\mathbf{A p}(x, C)$ through the MzScheme sentence: (applicability-vectors $M_{x} D$ ). The procedure works as follows: 
1. It starts defining locally:

- The node-structure as $<$ node $>::=[m ; V]$, where $m$ is a multiset, and $V=\left[v_{1}, v_{2}, \ldots, v_{s_{x}}\right]$.

- The final-node? test. A node $n=[m ; V]$ is a final node if $\forall d_{r} \in$ $D\left(d_{r}=\# \mathbf{f} \vee m<d_{r}\right)$.

- The generate-op function. It returns the operators list $\left(d_{1}, d_{2}, \ldots, d_{s_{x}}\right)$.

- The apply-op procedure. From a node $n=[m ; V]$, and an operator $d_{r} \neq \# \mathrm{f}$, it returns the successor node $n^{\prime}=\left[m^{\prime} ; V^{\prime}\right]$, with $m^{\prime}=m-$ $d_{r}, v_{r}^{\prime}=v_{r}+1$, and $v_{j}^{\prime}=v_{j}, \forall j \neq r$. If $d_{r}=\# \mathrm{f}$, then it returns \#f.

2. Then, it builds the init-node: $n_{0}=\left[M_{x} ;\left[\begin{array}{lll}0,0, & s_{x} & ,\end{array}\right]\left[\begin{array}{l}\text {. } \\ \text {. }\end{array}\right.\right.$

3 . It expands the tree through the sentence:

(breadth-expansion-tree

init-node final-node? generate-op apply-op)y

4. Finally, it returns the applicability vector $V$ of every final node $[\mathrm{m} ; \mathrm{V}]$.

Procedure applicability-vectors $\left(M_{x} D\right)$

1. Local definitions

$$
\begin{aligned}
<\text { node }>\quad::= & {[m ; V] \quad ; \text { whith } V=\left[v_{1}, v_{2}, \ldots, v_{s_{x}}\right] } \\
\text { final }- \text { node? }::= & \lambda_{1}:<\text { node }>\longrightarrow\{\# \mathrm{t}, \# \mathrm{f}\} \\
\text { generate }- \text { op }::= & \lambda_{2}:<\text { node }>\longrightarrow\left(d_{1}, d_{2}, \ldots, d_{s_{x}}\right) \\
\text { apply }- \text { op }::= & \lambda_{3}: r \times<\text { node }>\longrightarrow\left[m^{\prime} ; V^{\prime}\right] \\
& \text { with, } m^{\prime}=m-d_{r}, V^{\prime}=\left[v_{1}^{\prime}, v_{2}^{\prime}, \ldots, v_{s_{x}}^{\prime}\right], \\
& \text { being, } v_{r}^{\prime}=v_{r}+1 \text { but, } v_{j}^{\prime}=v_{j} \forall j \neq r
\end{aligned}
$$

2. The initial node

$$
\begin{aligned}
& m_{0} \leftarrow M_{x} \\
& V_{0} \leftarrow\left[0,0, s_{x} ., 0\right] \\
& n_{0} \leftarrow\left[m_{0} ; V_{0}\right]
\end{aligned}
$$

3. The final-nodes

final-nodes $\leftarrow$ (breadth-expansion-tree

$n_{0}$ final-node? generate-op apply-op)

4. Returns the vector $V$ of every node $[\mathrm{m} ; \mathrm{V}]$ of final-nodes

Notes:

- Every $v_{r}$ counts the times the rule $r$ could be applied.

$-\lambda_{1}([m ; V])=\# \mathrm{t} \leftrightarrow \forall d_{r} \in D\left(d_{r}=\# \mathbf{f} \vee m<d_{r}\right)$

$-\lambda_{3}(r,[m ; V])=\# \mathbf{f}$ if $d_{r}=\# \mathbf{f}$

\subsection{The tag-rules procedure}

The tag-rules procedure updates the app-tag $\alpha_{r}$ of those rules $r$ of $R^{*}$ that are applicable to a given configuration $C=(\mu, M)$. The MzSheme sentence (tag-rules $C R^{*} \rho$ ) returns $R^{C}$. The procedure works as follows: 
1. It starts getting the degree, $N$, of $\Pi$.

2. Then, its work is based on an external and an internal loop, to go through the membranes and through the rules of every membrane, respectively.

- The external loop generates $R_{x}^{C}$, for every $x=1,2, \ldots, N$, and, once it is finished, it builds $R^{C}=\left(R_{1}^{C}, R_{2}^{C}, \ldots, R_{N}^{C}\right)$. If $x \notin V(\mu) \vee M_{x}=\emptyset$, then $R_{x}^{C}=R_{x}^{*}$, otherwise, $R_{x}^{C}$ has to be generated by the internal loop.

- The internal loop generates $R_{x}^{C}$ for a given $x \in V(\mu)$. It checks the applicability of every rule $r_{x, j} \in R_{x}^{*}$ to $C$, it changes $\alpha_{x, j}$ from \#f to \#t if so, and it obtains the tagged-rule $r_{x, j}^{C}$; finally, it builds and returns to the external loop, $R_{x}^{C}=\left(r_{x, 1}^{C}, r_{x, 2}^{C}, \ldots, r_{x, s_{x}}^{C}\right)$.

3. It returns $R^{C}$.

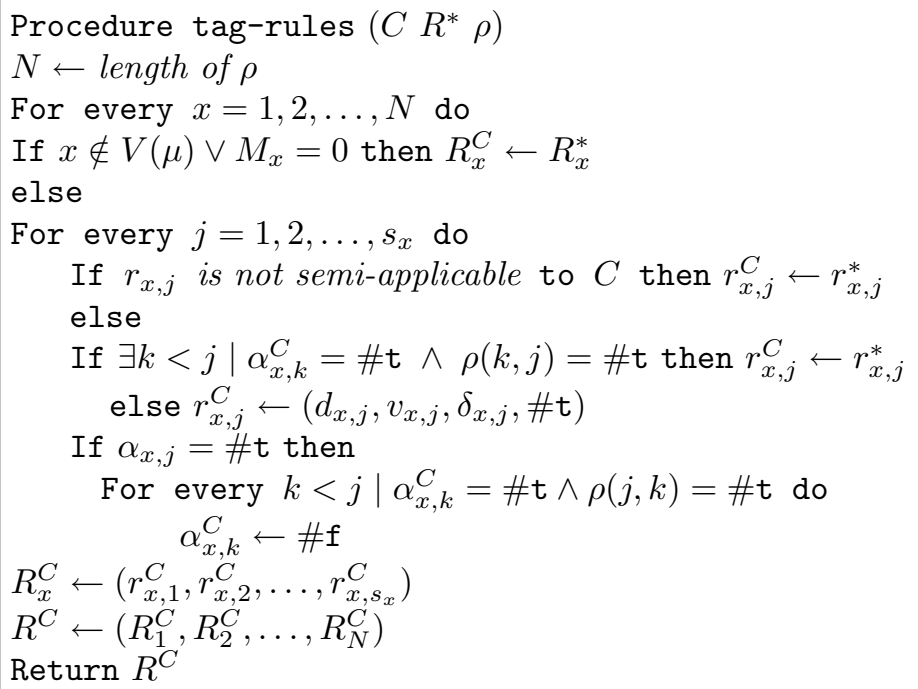

\subsection{The apply-matrix procedure}

The apply-matrix procedure computes one transition step, $C^{\prime}=P(C)$, from a configuration, $C=(\mu, M)$, and an applicability matrix, $P \in \mathbf{M}_{\mathbf{A p}}(C)$. The MzSheme sentence is (apply-matrix $C P R^{C}$ ). It works in two steps:

1. For every membrane $x$ in $C$ and every rule $r_{x, j}$ in $R_{x}^{C}$, provided $P_{x, j} \neq 0$ :

- $r_{x, j}$ is applied $P_{x, j}$ times without dissolution. So, some objects of membrane $x$ are consumed, and maybe itself and/or, its father and children receive some objects. A more internal loop identifies the target where every action of the rule sends its objects,

- then, if $r_{x, j}$ is a dissolution rule, $x$ is stored in $\Delta$ as a node to be dissolved. 
2. Then, we visit the nodes of $\mu$ in a bottom-up ordered way, the nodes kept on $\Delta$ are dissolved. Every dissolved node sends its objects (and children) to its father and disappears from $\mu$.

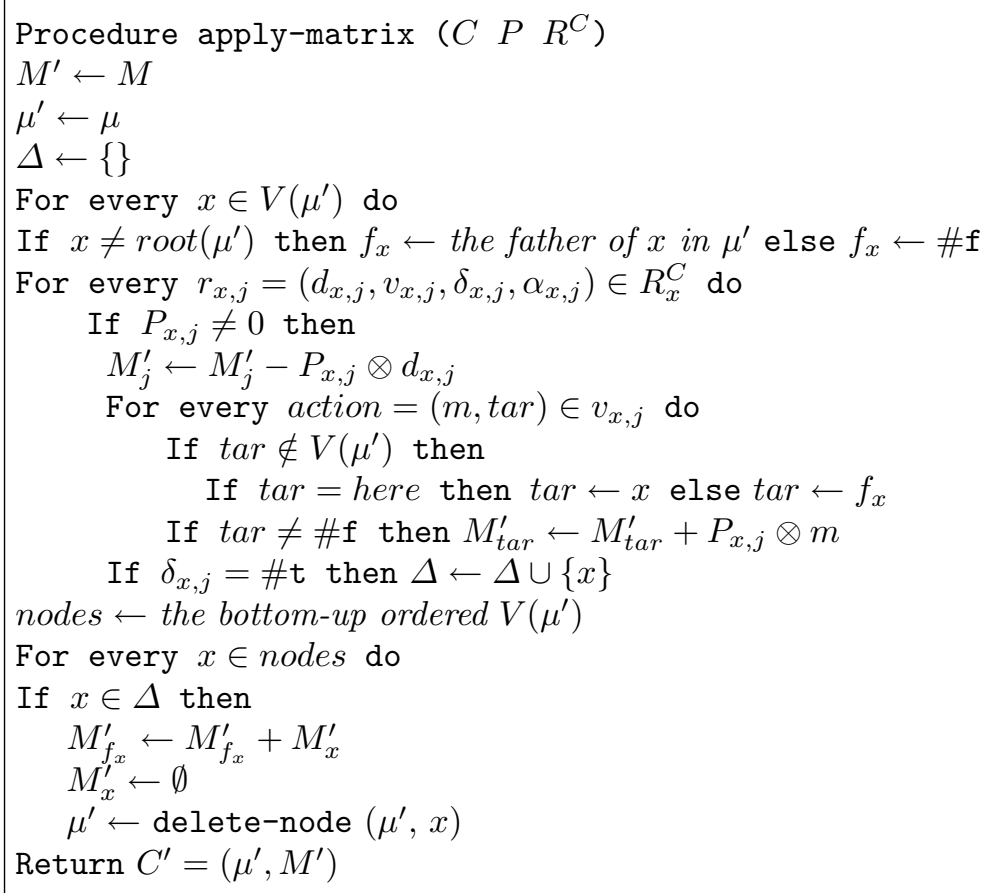

\section{A complete example: Generating squares $1^{2}, 2^{2}, \ldots, n^{2}$}

Finally we present here a complete example to illustrate the way our simulator should be used. The $\mathrm{P}$ system to be considered is the following one:

\subsection{The input data}

First of all, we have to input the data describing the $\mathrm{P}$ system. We do that defining the different elements: $A, N$, struct, output, objects, rules, and orders. As we need the symbol $a^{n} c f$, for the given $n$, this one is generated by the auxiliar procedure generate-symbol. The MzScheme sentence (sq1 $n$ ), assigns the respective value to every compound, and invokes the parser/compiler. 


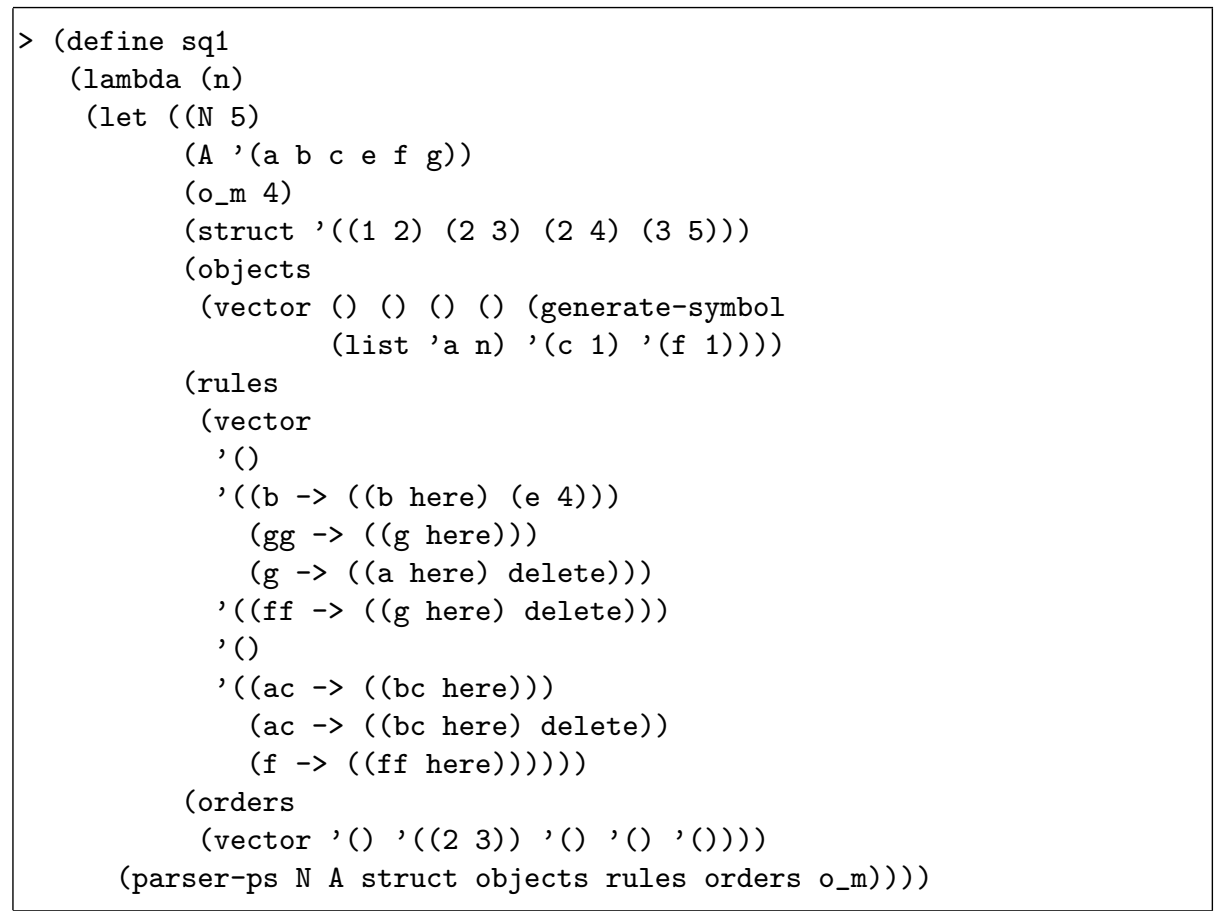

\subsection{The parser-compiler}

The parser/compiler returns the internal representation of the $\mathrm{P}$ system, and displays it in a readable way. So, if $n=4$ the sentence

(define ps (sq1 4))

defines, if no error occurs, ps as the representation to be used with the configurations procedure.

\subsection{Configurations}

Finally, using the procedure configurations to expand the computation tree, we obtain all configurations until the given level. In particular, with an appropriate level we get all the final configurations. In the previous example it is enough to use 9 as depth level. 


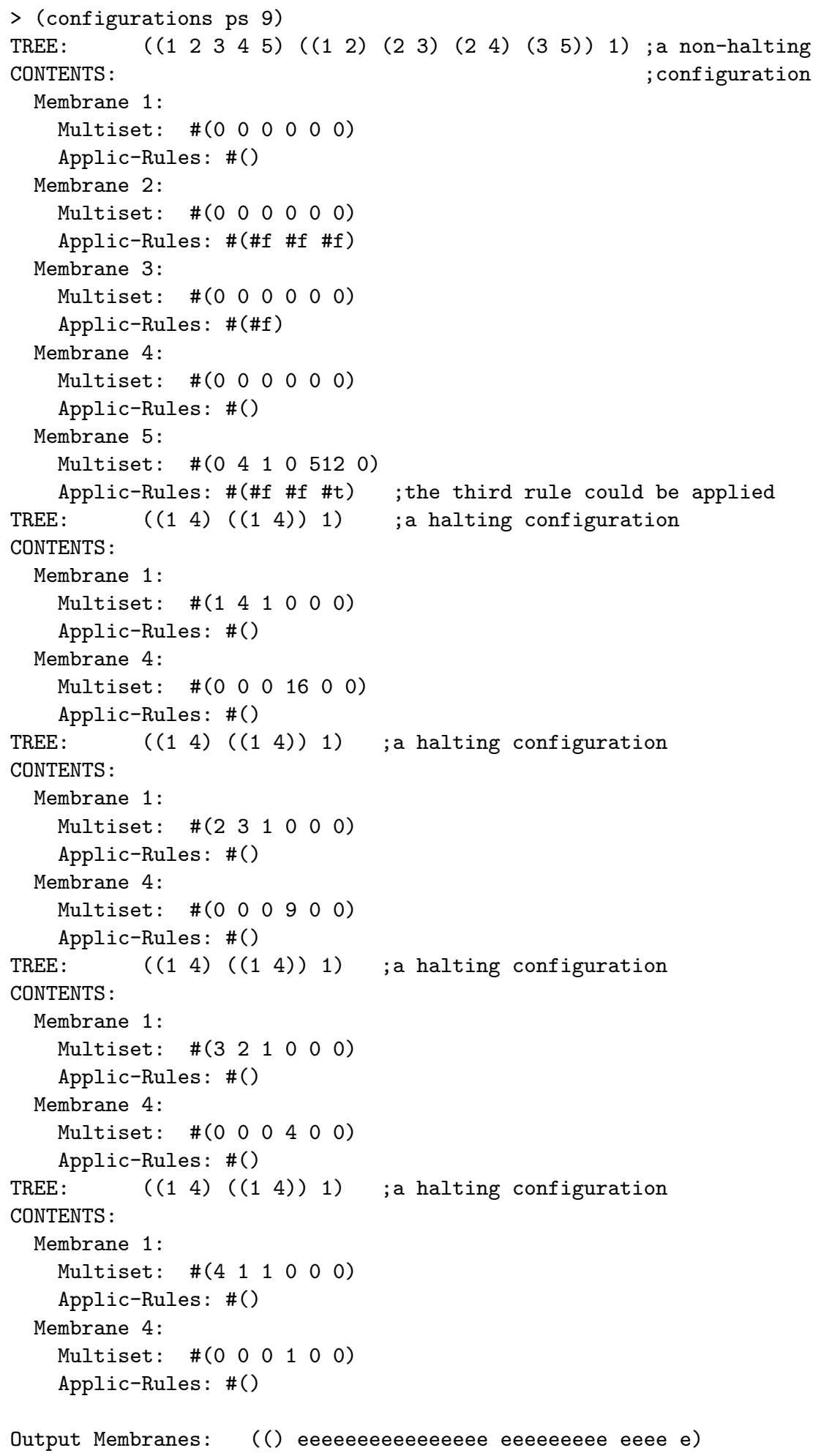

Output Membranes: ( ( $)$ eeeeeeeeeeeeeee eeeeeeeee eeee e) 


\section{Conclusions}

Up to now there is no implementation of $\mathrm{P}$ systems with a practical usefulness that allows the researchers to test and improve the abstract designs they make. The simulation of $\mathrm{P}$ systems by conventional programming languages can be considered not only as a practical approach to this computing model, but also as an useful way to understand and improved the $\mathrm{P}$ systems designed to solve real problems. We think that, because of the standard grammar it uses, the program presented here can be used both as a research tool and a teaching tool, allowing to see the way the $\mathrm{P}$ system evolves along its running. The program has been developed in such a way that it could be improved to simulate different variants of $\mathrm{P}$ systems. In a future work a graphical interface will be added, to make easier the interaction with the user.

\section{References}

1. Gh. Păun, Computing with membranes, Journal of Computer and System Sciences, 61, 1 (2000), 108-143, and Turku Center for Computer Science-TUCS Report No 208, 1998 (www.tucs.fi).

2. Gh. Păun, G. Rozenberg, A guide to membrane computing, Theoretical Computer Science, 287, 1 (2002), 73-100.

3. M.J. Pérez-Jiménez, F. Sancho-Caparrini. A formalization of transition P systems. Fundamenta Informaticae, 49, 1-3 (2002), 261-272.

4. M.J. Pérez-Jiménez, F. Sancho-Caparrini. Verifying a $P$ system generating squares. Romanian Journal of Information Science and Technology, 5, 2-3 (2002), 181-191.

5. MzScheme Home Page. http://www.cs.rice.edu/CS/PLT/packages/mzscheme/ 